\title{
Proceedings of the 2018 GRAPPA Collaborative Research Network Meeting
}

\author{
Deepak R. Jadon, Vinod Chandran, Alexis Ogdie ${ }^{(D)}$, Stephen R. Pennington, Carmel Stober, \\ Dafna D. Gladman (1), Maarten de Wit ${ }^{\mathbb{D}}$, Denis O’Sullivan, Philip Mease, April W. Armstrong, \\ Kristina Callis Duffin, Conor Magee, Philip Helliwell, Christopher T. Ritchlin, \\ and Oliver FitzGerald
}

\begin{abstract}
The Group for Research and Assessment of Psoriasis and Psoriatic Arthritis (GRAPPA)-Collaborative Research Network (CRN) intends to launch and secure funding for 3 pilot projects related to psoriatic disease, psoriatic arthritis (PsA), and cutaneous psoriasis (PsC). The first pilot project, a PsA Biomarkers for Joint Damage (BioDAM) pilot, will seek to determine the independent predictive ability of serum biomarkers for joint damage in PsA. The second pilot project will aim to identify predictors of the development of PsA among patients with PsC. The third pilot project will aim to identify biomarkers that predict treatment response in PsA and PsC. These pilot projects will prompt the development of clinical protocols to operate across participating centers, lead to the development of standard operating procedures for the collection and transport of biosamples across international borders, and begin to establish administrative and managerial structures for the CRN. The CRN hopes that the successful completion and research outputs of these 3 pilot projects will demonstrate the CRN's value to prospective collaborators and sponsors and thereby secure sustainable longterm funding. (J Rheumatol Suppl. 2019 June;95:11-19; doi:10.3899/jrheum.190118)
\end{abstract}

Key Indexing Terms:

PSORIATIC ARTHRITIS

PSORIASIS
OUTCOME MEASURES

RESEARCH

BIOMARKERS

GRAPPA

From the Rheumatology Research Unit, Cambridge University Hospitals National Health Service (NHS) Foundation Trust, Cambridge; Leeds

Institute of Rheumatic and Musculoskeletal Medicine, University of Leeds, Leeds; Bradford Hospitals NHS Foundation Trust, Bradford, UK;

Department of Medicine, Division of Rheumatology, University of Toronto; Krembil Research Institute, Toronto Western Hospital, Toronto, Ontario,

Canada; Perelman School of Medicine, University of Pennsylvania, Philadelphia, Pennsylvania; Swedish Medical Center/Providence-St. Joseph Health, Seattle, Washington; University of Southern California, Los Angeles, California; University of Utah, Salt Lake City, Utah; Division of Allergy, Immunology, and Rheumatology, University of Rochester Medical Center, Rochester, New York, USA; Conway Institute for Biomolecular Research, University College Dublin; Our Lady's Hospice and Care Services; Department of Rheumatology, St. Vincent's University Hospital, Dublin, Ireland; Department of Medical Humanities, Amsterdam University Medical Center, Amsterdam, the Netherlands.

As part of the supplement series GRAPPA 2018, this report was reviewed internally and approved by the Guest Editors for integrity, accuracy, and consistency with scientific and ethical standards.

D.R. Jadon, MBBCh, MRCP, PhD, Director of Rheumatology Research Unit, Cambridge University Hospitals NHS Foundation Trust;

$V$. Chandran, MBBS, MD, DM, PhD, Department of Medicine, Division of Rheumatology, University of Toronto, Toronto Western Hospital, Krembil Research Institute; A. Ogdie, MD, Assistant Professor of Medicine and Epidemiology, Perelman School of Medicine, University of Pennsylvania; S.R. Pennington, Professor of Proteomics, PhD, Conway Institute for Biomolecular Research, University College Dublin; C. Stober, PhD, $M B C h B, M R C P$, Consultant Rheumatologist, Cambridge University Hospitals NHS Foundation Trust; D.D. Gladman, MD, FRCPC, Professor of Medicine, University of Toronto, and Senior Scientist, Krembil Research Institute, Toronto Western Hospital; M. de Wit, PhD, Patient Research Partner, Department of Medical Humanities, Amsterdam University
Medical Center; D. O'Sullivan, BE, Patient Research Partner, Our Lady's Hospice and Care Services; P.J. Mease, MD, Rheumatology Research, Swedish Medical Center/Providence-St. Joseph Health; A.W. Armstrong, MD, MPH, University of Southern California; K. Callis Duffin, MD, University of Utah; C. Magee, $M B, B A O, B C h$, St. Vincent's University Hospital; P.S. Helliwell, DM, PhD, FRCP, Leeds Institute of Rheumatic and Musculoskeletal Medicine, University of Leeds, and Bradford Hospitals NHS Foundation Trust; C.T. Ritchlin, MD, MPH, Professor of Medicine, Division of Allergy, Immunology, and Rheumatology, University of Rochester Medical Center; O. FitzGerald, MD, FRCPI, FRCP(UK), Newman Clinical Research Professor, Department of Rheumatology, St. Vincent's University Hospital and Conway Institute for Biomolecular Research, University College Dublin. Professor O. FitzGerald and Professor C.T. Ritchlin are joint senior authors.

Address correspondence to Dr. D.R. Jadon, Director of the Rheumatology Research Unit, Cambridge University Hospitals NHSFT, Addenbrooke's Hospital, Hills Road, Cambridge, CB2 OQQ, UK.

E-mail:dj351@medschl.cam.ac.uk

The Group for Research and Assessment of Psoriasis and Psoriatic Arthritis (GRAPPA)-Collaborative Research Network (CRN) held its second meeting at the GRAPPA 2018 annual meeting in Toronto, Ontario, Canada. The CRN meeting was organized by a committee co-chaired by Professors Oliver FitzGerald and Christopher Ritchlin. The meeting was attended by 28 rheumatologists, 3 dermatologists, 6 patient research partners (PRP), 1 non-medical scientist, and 14 people from the pharmaceutical industry.

The meeting's objectives were to discuss launching and

Personal non-commercial use only. The Journal of Rheumatology Copyright (c) 2019. All rights reserved. 
securing funding for 3 pilot projects under the auspices of the GRAPPA-CRN. These pilots would (1) prompt the development of clinical protocols to operate across participating centers; (2) lead to the development of standard operating procedures (SOP) for the collection, storage, and transport of biosamples; (3) begin to establish administrative and managerial structures for the $\mathrm{CRN}$; and (4) ultimately demonstrate the CRN's value, which would encourage new centers to contribute and new sponsors to support the CRN. The longterm goal of securing more sustainable funding was also discussed and was inspired by the Accelerating Medicines Partnership (AMP) model ${ }^{1}$.

\section{Collaborative Research in Inflammatory Arthritis: Lessons from AMP}

Professor Vivian Bykerk presented a keynote talk that described AMP ${ }^{1}$, its successes, and the lessons learned over the past 4 years. AMP has been a platform for clinical, translational, and implementational science that was created to resolve important clinical-practice challenges in systemic lupus erythematosus (SLE), rheumatoid arthritis (RA), type 2 diabetes, and Alzheimer disease. AMP has worked closely with research groups in the United Kingdom and the European Synovitis Study Group to enable ultrasound (US)-guided synovial tissue biopsies from rheumatoid joints. These technologies are cutting edge and include robust high-resolution sequencing and transcriptomic profiling at the single cell level ${ }^{1,2}$. The introduction of these new technologies has brought new skills and expertise to the contributing centers, which will be valuable for research projects outside the AMP.

Professor Bykerk briefly described the past 4 years of the AMP process. In the first year, the initial request for applications for proposals related to rheumatologic diseases was made for sites that could provide studies or technologies to advance precision medicine in RA and SLE. There were 5 sites selected for RA and 5 sites selected for SLE. Collaborators trained healthcare providers to perform US-guided synovial biopsies at these sites. The SLE studies focused on the analysis of renal biopsies.

In the second year, the group created a network study, which was a milestone-driven investigative period for the development and validation of SOP and protocols with 3 key phases: SOP development, the implementation of pipeline analytics that compared RA with osteoarthritis, and the development of an observational study of RA. AMP is structured with the following 10 committees: (1) executive; (2) steering; (3) national leadership committee (NLC; reporting to the steering committee); (4) disease focus group for RA and SLE (reporting to the NLC); (5) clinical study group; (6) technology; (7) tissue group; (8) policy; (9) operations; and (10) fibroblast, monocyte, $\mathrm{T}$ cell, and $\mathrm{B}$ cell group. Committees were designed to be small to permit timely decisions, and the US National Institutes of Health (NIH) provide overarching administration. Each AMP center has a principal investigator (PI) who sits on the NLC and has 1 vote (not all PI are on the steering committee).

Methods to devise, validate, and establish SOP for tissue sampling, processing, and analysis were described. Pipeline analytics have included the use of CyTOF mass cytometry, bulk RNA sequencing, single-cell RNA sequencing, multiparameter flow cytometry, and histomorphic studies that use pathway analysis. Nested studies have permitted the investigation of novel techniques such as tetramer-based sorting of antigen-specific lymphocyte, $\mathrm{T}$ and $\mathrm{B}$ cell receptor sequencing, and single-cell laser-capture microscopy. It was emphasized that expertise in systems biology analysis has been extremely important. New techniques have also been developed, including Dropseq, which is used to sequence arthroplasty tissue at the bedside ${ }^{2}$; 10X-genomics; and CITE-seq, which permits the cellular indexing of transcriptome and epitopes by sequencing, thus combining the identification of single-cell transcriptomes and protein markers at the single-cell level ${ }^{3}$.

AMP is now entering phase 2, an observational study of patients with active RA (Clinical Disease Activity Index $>10$ ) derived from 1 of 3 groups: disease-modifying antirheumatic drug (DMARD)-naive or minimally exposed, methotrexate (MTX)-inadequate responder at Week 12, and tumor necrosis factor (TNF) inhibitor-inadequate responders. US-guided synovial biopsies have been taken in 48 patients (knee $40 \%$, wrist $42 \%$, ankle $10 \%$, metacarpophalangeal joint $8 \%$, and other sites) across 20 trained centers. In phase 2, 150 subjects are being sought, with 20 having repeat biopsies.

Funding of US\$ 3 million was secured to establish the cohort, to do the clinical phenotyping, and to perform biopsies. A further US\$ 3 million was secured to perform the analyses. Funding has been derived primarily (40\%) from the pharmaceutical industry (AbbVie, Bristol-Myers Squibb, Merck, Pfizer, Sanofi, and Takeda), followed by the Foundation for the NIH (40\%) and other non-profit organizations (10-20\%; e.g., Arthritis Foundation, Alliance for Lupus Research, and the Rheumatology Research Council).

The AMP group initially met very frequently to gain consensus on strategic plans. It continues to meet regularly to update contributors on progress, to draft publications, and to raise enthusiasm among researchers. Operationally, all synovial biopsy samples are transported to 1 center for analysis. The nested studies were designed to be longitudinal rather than cross-sectional, to improve the generalizability of the results. Deciding on the SOP was mostly determined by the study question being posed. Of note, no issues were encountered in transferring biopsy samples between the United States and the United Kingdom. Clinical data are entered into an online database (REDCap) and supervised by Stanford University, but as with all studies, some missing data remain a challenge. AMP has inspired groups of independent investigators to work more closely as part of a consortium.

Personal non-commercial use only. The Journal of Rheumatology Copyright $\odot$ 2019. All rights reserved 


\section{CRN Pilot Study 1: PsA-BioDAM}

Dr. Vinod Chandran acted as a convener to propose a PsA-Biomarkers for Joint Damage (BioDAM)-related CRN pilot study. Dr. Chandran was supported by Dr. Walter Maksymowych, Professor Oliver FitzGerald, Dr. Philip Mease, Heidi Bertheusen (PRP), Maarten de Wit (PRP), and David Collier (Amgen).

Given that PsA is a heterogeneous disease with erosions evident in $47 \%$ of cases by 2 years, there is a need to identify biomarkers to help stratify treatment and identify changes in disease activity with treatment. Known clinical prognostic markers include polyarticular disease, dactylitis, high acute-phase response, and delayed presentation to specialist rheumatology care. Joint erosions and damage are markers of severe disease and have previously been demonstrated to be associated with poorer functional status, worse economic effect, and a risk factor for mortality.

The PsA-BioDAM project was developed with the primary objective to determine the independent predictive ability of serum biomarkers for joint damage. Candidate biomarkers include collagen proteins, serum calgranulin (S100A8/S100A9), macrophage colony-stimulating factor, receptor activator of nuclear factor- $\kappa \mathrm{B}$ (but not Dickkopf-1), vascular endothelial growth factor, angiopoietin-2, and serum amyloid A. Advanced discussions have taken place between GRAPPA and Amgen on using the SEAM study [phase III, 48-week study comparing etanercept (ETN) and MTX as mono- and combination-therapy in subjects with PsA; radiographs performed at 6 and 12 months; serum sampled at baseline and 8,24, and 48 weeks] biosamples as part of this PsA-BioDAM-related CRN pilot study.

A leading hypothesis is that surrogate biomarkers will predict which patients are at risk for peripheral radiographic damage in PsA. The aim of the study will be to identify biomarkers that predict joint damage with the goal to stratify treatment in the early stages and limit bone resorption and cartilage degradation. The primary study objective will be to determine the independent predictive validity of several biomarkers in predicting structural damage (erosions) in patients with PsA who receive MTX, ETN, or both. An aliquot of each serum sample with the linked clinical and radiographic data will be provided to GRAPPA. A panel of over 200 candidate protein biomarkers will be tested on the serum samples that are provided using optimized multiple reaction monitoring (MRM) assays and testing for metalloproteinase inhibitor 1, $\alpha 1$-acid glycoprotein 2, serotransferrin, platelet glycoprotein Ib $\alpha$ chain, di-N-acetyl chitobiase, gelsolin, fibroblast growth factor receptor 1 , leukocyte immunoglobulin-like receptor subfamily B member 2, C-X-C motif chemokine 13, ADP-ribosyl cyclase 2, complement component $\mathrm{C} 7$, haptoglobin-related protein, apolipoprotein E, apolipoprotein D, GTPase KRAS, complement factor $\beta$, TNF- $\alpha$, SPARC, C-X-C motif chemokine $10, \alpha 1$-antichymotrypsin, and tyrosine-protein phospha- tase nonreceptor type 2. It is anticipated that a panel of markers with baseline or change values will be predictive. Discussions are in progress to determine who should perform the analyses: academics, industry scientists, or a combined effort. It was emphasized that the PsA-BioDAM study aims to identify serum biomarkers that predict radiographic damage that are independent of $\mathrm{C}$-reactive protein to add value to known biomarkers.

The model agreement is now also being discussed with Lilly (SPIRIT-P1 and P2 studies), Pfizer (OPAL Broaden and Beyond studies), AbbVie (Upadacitinib studies), and Bristol-Myers Squibb (986165 TYK2i studies) as providers of serum samples, which will enable this study to be a low-risk, lower-cost pilot for the CRN.

\section{CRN Pilot Study 2: Predicting the Development of PsA Among Patients with Psoriasis}

Drs. April Armstrong and Alexis Ogdie co-led the proposal for another CRN pilot study related to predicting the development of PsA among patients with cutaneous psoriasis only (PsC). Drs. Armstrong and Ogdie were supported by Professors Christopher Ritchlin, Dafna D. Gladman, Steve Pennington, Philip Helliwell, and Cheryl Rosen, and Drs. Carmel Stober, Jose Scher, Souyma Chakravaty (Janssen), Jaci Anderson (AbbVie), John Latella (PRP), Jeffrey Chau (PRP), and Rodrigo Firmino (PRP).

Dr. Stober described how the evidence to date suggests that the progression of PsC to PsA is determined by the complex interplay of genes, microbiome, immune system, environment, and other exposures. In RA, it has been demonstrated that genes in the MHC region, as well as PTPN22, $P A D I 4$, and CTLA-4, contribute to genetic susceptibility for RA. Genome-wide association studies indicate that genetics account for $36-65 \%$ of RA heritability, with the shared epitope being a major contributor ${ }^{4}$. Several transcriptome and proteomic studies have identified differential expression of RNA and proteins in RA ${ }^{5}$.

Dr. Ogdie gave an epidemiological perspective on the various types of evidence in literature, including associations (from cross-sectional or case-control studies), etiologic risk factors (which have a temporal association that occurs before the disease has begun and that may lead to the progression of PsC to PsA), and predictors including PsC factors that might identify which patients are likely to have PsA but may or may not be a part of the causal pathway, as shown in Figure 1. Subclinical changes in the entheses and joints have been investigated in $\mathrm{PsC}$ cases using US, magnetic resonance imaging, positron emission tomography, and microcomputed tomography imaging to ascertain whether they predict progression to PsA. However, there is limited prospective data and results are heterogeneous ${ }^{6,7}$. Ongoing work will aim to define these earlier stages of disease.

Dr. Rosen gave an overview of the University of Toronto Psoriasis Cohort, a cohort of patients with PsC who have

Personal non-commercial use only. The Journal of Rheumatology Copyright $@$ C 2019. All rights reserved. 


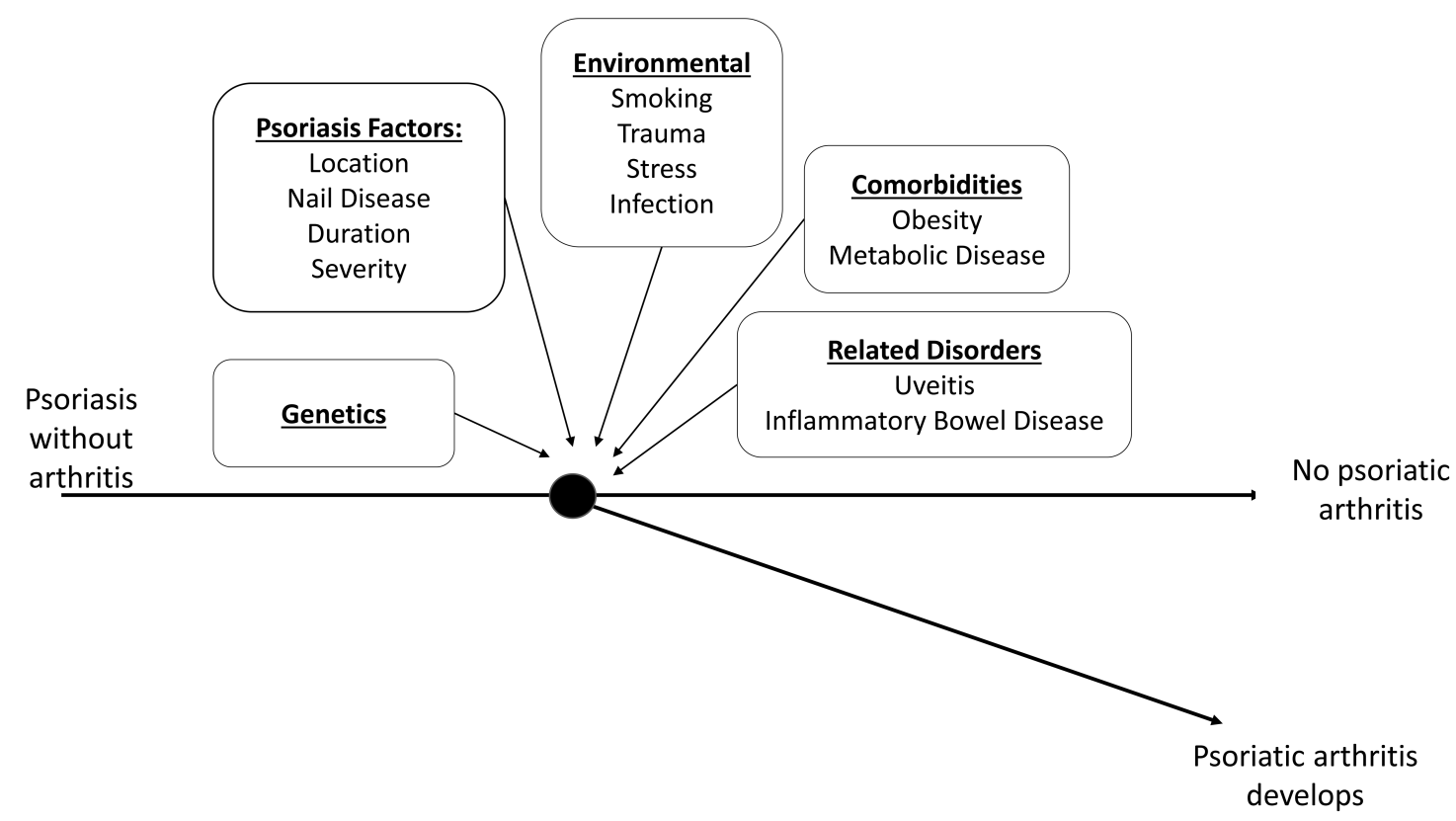

Figure 1. Epidemiological perspective on the interplay of risk factors, associations, and psoriasis factors that might contribute to the progression of cutaneous psoriasis to psoriatic arthritis.

been followed at least yearly since 2006 to identify factors associated with the development of PsA. At 8 years of followup, 51 of 464 enrolled PsC cases had developed PsA, which equates to an annual incidence rate of 2.7 cases per 100 patients. Severe psoriasis, psoriatic nail pitting, uveitis, and a family history of $\mathrm{PsA}^{8}$ were associated with an increased risk of developing PsA. The risk of new-onset PsA remained the same and did not lessen over time. Of note, there appears to be a phase prior to the diagnosis of PsA of increased nonspecific musculoskeletal symptoms ${ }^{8}$. In other studies, obesity has been demonstrated to be a risk factor for the progression of $\mathrm{PsC}$ to $\mathrm{PsA}^{9,10}$, but in some respects is a confounder, particularly in the relationship between psoriasis severity and the development of PsA. Another unique "predictor" that was discussed is smoking. In the Toronto cohort, 1 study found current smoking was associated with a decreased likelihood of developing PsA. However, because smoking is also associated with the development of PsC, this may be a misleading finding, because when compared to the general population, smoking either is not associated with the development of PsA or is positively associated. Thus, comparing potential risk factors between populations with PsC and PsA, rather than comparing populations with PsA and controls (particularly when the factor may trigger the development of $\mathrm{PsC}$ ), may cause spurious results. It is therefore important to be aware of collider stratification bias $^{11}$.

Drs. Scher and Pennington described the pathophysiology, varying phenotypes, and candidate biomarkers of PsC progressing to PsA. They emphasized that the onset of PsA should not be considered in a dichotomous manner but rather as a spectrum of increasing manifestations. In some respects, clinicians and researchers create arbitrary thresholds for when a diagnosis of PsA is justified, and this pitfall should be avoided to identify a biomarker of PsC progressing to PsA. A variety of other approaches were proposed, including leveraging skin phenotypes as biomarkers and considering different biomarkers for transition into various domains. It was debated whether the PsA research community is in a position to generate SOP for extraction and processing on different platforms, as well as whether data from various processing centers can be combined. The need for "big data" analysis methods to manage this was again emphasized.

Professor FitzGerald described how HLA genotype modulates disease expression in PsA, the need for dense genotyping of immune-related susceptibility loci, and the emerging and compelling evidence for the existence of PsA-specific risk loci $^{12}$. Of note, HLA-B27 is the only validated biomarker of $\mathrm{PsC}$ progression to PsA. Some oncology researchers are moving away from genomics and using RNA sequencing and proteomics to identify biomarkers in similar contexts. There were 3 key discussion items debated:

1. whether susceptibility loci identified as being specific for PsA are sufficiently sensitive and/or specific to discriminate those who may develop PsA from those with $\mathrm{PsC}$;

2. whether the current PsC cohorts (e.g., Toronto, Frankfurt, United Kingdom, and Dublin) are large enough to test the ability of PsA-specific loci and whether they are collecting appropriate clinical data, imaging, and biosamples; and

Personal non-commercial use only. The Journal of Rheumatology Copyright $\odot$ 2019. All rights reserved 
3. whether future studies should include the genomewide investigation of transcription factors in CD8+ memory T cells in both PsA and PsC.

\section{CRN Pilot Study 3: Biomarkers Predicting Treatment Response in PsA and PsC}

Professor FitzGerald convened the third pilot study and was supported by Professors Dafna D. Gladman and Philip Mease, and Drs. Deepak Jadon, Kristina Callis Duffin, Stefan Siebert, Conor Magee, Niti Goel, and Denis O'Sullivan (PRP), Shelly Kafka (Janssen), Lara Fallon (Pfizer), and Maureen Kelly (AbbVie).

Dr. Mease and Denis O'Sullivan introduced the major unmet need for biomarkers to predict treatment response in PsA and PsC. They discussed how lessons can be learned from oncology, where disease management is now molecularly guided to treatments that are less likely to be toxic and more likely to be efficacious, which will ultimately improve clinical outcomes. This "personalized" and "precise" approach, which is tailored to the individual patient's genetic, epigenetic, cellular, and/or molecular phenotype, is needed in psoriatic disease management. In some respects, the current treatment paradigm in psoriatic disease is comprised of (1) stepwise treatment that starts with lower cost, potentially less toxic but also potentially less effective medicines; (2) "trial and error" treatment choices that often start with MTX and then possibly add or substitute with another conventional synthetic DMARD (csDMARD); and (3) treatment that tries 1 or 2 anti-TNF therapies. These are often followed by treatment with antagonize interleukin (IL)-17, IL-12/23, phosphodiesterase 4, or Janus kinase without much information to guide that decision.

While imprecise choices may not be fatal, as in oncology, uncontrolled symptoms, progressive structural damage, work loss and disability, toxicity, and treatment expense all represent a "cost" to the patient, the patient's family, and society. A precision medicine approach in psoriatic disease may lead to rational stepwise and/or combination therapy; quicker achievement and maintenance of treat-to-target goals of remission or low disease activity; better prediction of adverse effect profile; reduced cost in terms of toxicity, structural damage, work productivity, and economics; and "guided treatment regimens" of induction, maintenance, tapering, or withdrawal. Miyagawa, et al have recently demonstrated that response to TNFi, IL-17i, and IL-12/23i therapies in PsA can be associated with the relative abundance of Th1 and Th17 cells in the peripheral blood as detected by flow cytometry-based immunophenotyping ${ }^{13}$.

Drs. Magee and Jadon presented preliminary results of their systematic literature review (SLR) on biomarker (genetic, serum, cellular, urine, synovial, and skin biopsy) predictors of treatment response in PsA and PsC. Of 558 articles retrieved, 31 met eligibility criteria: 11 PsA and 20 PsC. In PsA, 3 studies examined synovium, 1 examined genetic polymorphism, and 7 examined serum proteins. In PsC, 8 studies examined genetic polymorphisms, 2 examined skin biopsies, and 10 examined serum or plasma proteins or antibody levels. There was much heterogeneity in study designs, biosampling methods, analysis techniques, and clinical outcome measures. Metaanalysis was therefore not possible. Of note, few biomarkers were tested in independent cohorts, and there are, therefore, no validated or even strong candidate biomarkers of treatment response in either PsA or PsC. The full SLR will be reported in due course.

Dr. Jadon described ongoing but unpublished research on this theme. The Dublin Measuring Outcome in Psoriatic Arthritis group (MOPsA; led by Professor FitzGerald) is analyzing the Tight Control Works in Psoriatic Arthritis cohort (TICOPA; led by Drs. Helliwell and Coates; $n=82$ baseline PsA samples) to identify proteins that predict achieving minimal disease activity (MDA). In addition, PsA samples from Amsterdam from patients of Drs. Leonieke van Mens and Dominique Baeten who are starting golimumab or MTX are undergoing MRM assay to test for predictors of treatment response. A trial within a cohort study (MONITOR) of 3 centers in the United Kingdom (led by Drs. Coates, Jadon, and William Tillett) is now recruiting to an inception cohort of patients with PsA $(n=500)$ followed for 5 years, with whole blood (cellular, DNA, RNA, serum), urine, and stool collected at first presentation and at 3, 6, 9, 12, 18, 24, 36, 48, and 60 months. Patients will be treated as standard practice with csDMARD, biologic DMARD, and targeted synthetic DMARD. Predictors of treatment response will be 1 of several study endpoints. Dr. Stefan Seibert and Professor Iain McInnes in Glasgow are assessing several biomarkers of therapy from RA in patients with PsA to determine generic biomarkers of response, rather than drug- or disease-specific biomarkers, as part of the ScOttish Psoriatic arthritis Observational Study (SOPHOS) and Biomarkers Association in Skin and Synovium in PsA (BASSPA) study. Psoriatic Arthritis Research Consortium (PARC) is a 4-center collaboration on the east coast of the United States (led by Drs. Ogdie, Scher, Soumya Reddy, and Jessica Walsh) that is longitudinally collecting clinical, imaging, and biosamples in PsA cases.

Professors FitzGerald and Gladman presented how this pilot may be taken forward with GRAPPA alone or through collaboration with industry partners. Considerations for a collaborative project with industry might include working with 1 or more companies that have conducted randomized controlled trials (RCT) with detailed baseline and followup measures that have stored biosamples (e.g., serum) that are then made available to GRAPPA researchers. An initial "discovery" type study would involve patients who clearly respond to the study drug (either MDA or remission criteria) who are then compared with patients who fail to meet a primary outcome measure (e.g., American College of Rheumatology $20 \%$ response criteria) or who get worse.

Personal non-commercial use only. The Journal of Rheumatology Copyright @ 2019 . All rights reserved. 
Baseline samples ( $\mathrm{n}=10-20$ per group) could be subjected to analysis using several platforms such as mass spectrometry-based protein analysis, SOMAscan, and Multiplex Luminex transcriptomics that look for individual or groups of markers that distinguish responders from nonresponders at baseline. The funding for such a study is not likely to be prohibitively expensive.

It was therefore proposed that 5 centers with a track record of clinical research, biosample collection, and biosample storage be identified. Then, 8 patients per center with active $\mathrm{PsC}$ and PsA who require treatment intervention (standard RCT entry criteria) are enrolled in an open-label study with an agreed therapy, likely starting with a TNFi, over a period of 6 months. Standard clinical data would be collected in an agreed database (likely REDCap) at baseline and at 3 and 6 months. Patients would be defined as responders if they meet either MDA or remission criteria, and nonresponders would be defined as those who fail to meet the primary outcome measure of American College of Rheumatology 20 or those who clinically deteriorate. The estimated number of responders, predicting that $25 \%$ reach MDA, is 10 . The estimated number of nonresponders, assuming a $30 \%$ nonresponse rate, is 12. Biosamples (e.g., blood, peripheral blood mononuclear cells, DNA, RNA, and possibly tissue) would be collected and stored at defined intervals using agreed SOP, transported to the iPART group, and then analyzed in 1 center using validated laboratory techniques. The baseline samples (discovery) could be subjected to analysis using several platforms that look for individual or groups of baseline markers that separate responders from nonresponders, such as liquid chromatograph ion spray mass spectroscopy, SOMAscan, Multiplex Luminex ELISA, and Transcriptomics. Identified biomarkers could be validated in all patients using assays such as MRM.

There are several advantages to such an approach. First, a small study would allow for clinical protocols to be developed by the CRN, which would operate across centers. Second, such an approach would allow for the development of SOP for the collection, storage, and transport of bio-samples across international borders. Third, this approach would ultimately provide evidence of the CRN's value and ability to share the clinical database and sample collection SOP with all centers that would like to collaborate and contribute.

Based on costs for similar clinical studies, it is estimated that the cost per patient enrolled would be CA $\$ 4800$. With the required 40 patients enrolled, the total cost would be CA $\$$ 192,000. This would include costs for database development and agreement; SOP development and agreement; and discovery and validation costs based upon samples being collected, stored, transported, and analyzed for 40 patients at 3 timepoints.

The next steps and timelines for this pilot are to (1) complete and publish the SLR (Quarter 4 of 2018);
(2) complete contracts with industry partners regarding the sharing of samples, funding, and work plan (Q4 2018); (3) identify industry partner(s) for assistance with pilot study development (Q1 2019); (4) prepare a detailed study proposal, including the development of a database, the standardization of SOP, and the development of ethics proposals (Q2 2019); and (5) identify 5 lead contributing centers (Q3 2019).

\section{DISCUSSION}

Prior to the 2018 GRAPPA annual meeting, the attendees were asked to review a draft of the "GRAPPA-CRN Strategic Plan." The entire GRAPPA membership then participated in 8 workshops at the annual meeting that were facilitated by members of the CRN steering committee. The draft strategic plan was amended based on comments received at the workshops and discussions at the subsequent $\mathrm{CRN}$ symposia. The final strategic plan for implementation is presented in Tables 1-5. Table 1 includes the executive summary and "elevator pitch" for the CRN. Table 2 describes the CRN's mission, vision, and goals and how these align with GRAPPA's broader mission. Table 3 details the CRN's strengths, weaknesses, opportunities, and threats analysis. Table 4 identifies the target stakeholders, landscape, and competition analysis. Finally, Table 5 details a 5 -phase plan, key team members, operational plan, financial projections, and implementation plan.

The CRN meeting provided an opportunity to identify 3 pilot projects, to discuss gaps in current knowledge of these themes, and to devise a research plan to address them. GRAPPA members heard from an expert from a similar large research consortium, which prompted the CRN to commence detailed planning of the 3 pilot research projects and their funding mechanisms.

\section{REFERENCES}

1. National Institutes of Health. Accelerating Medicines Partnership (AMP). Autoimmune diseases of rheumatoid arthritis and lupus. [Internet. Accessed December 22, 2018.] Available from: https://www.nih.gov/research-training/accelerating-medicinespartnership-amp/autoimmune-diseases-rheumatoid-arthritis-lupus

2. Rao DA, Gurish MF, Marshall JL, Slowikowski K, Fonseka CY, Liu Y, et al. Pathologically expanded peripheral T helper cell subset drives B cells in rheumatoid arthritis. Nature 2017;542:110-4.

3. Peterson VM, Zhang KX, Kumar N, Wong J, Li L, Wilson DC, et al. Multiplexed quantification of proteins and transcripts in single cells. Nat Biotechnol 2017;35:936-9.

4. Viatte S, Plant D, Raychaudhuri S. Genetics and epigenetics of rheumatoid arthritis. Nat Rev Rheumatol 2013;9:141-53.

5. Bakker MF, Cavet G, Jacobs JW, Bijlsma JW, Haney DJ, Shen Y, et al. Performance of a multi-biomarker score measuring rheumatoid arthritis disease activity in the CAMERA tight control study. Ann Rheum Dis 2012;71:1692-7.

6. Ash ZR, Tinazzi I, Gallego CC, Kwok C, Wilson C, Goodfield M, et al. Psoriasis patients with nail disease have a greater magnitude of underlying systemic subclinical enthesopathy than those with normal nails. Ann Rheum Dis 2012;71:553-6.

Personal non-commercial use only. The Journal of Rheumatology Copyright (C) 2019. All rights reserved. 
Table 1. CRN executive summary and "elevator pitch."

\section{The GRAPPA-CRN Strategic Plan}

1. Executive summary

- Significant advances in the treatment of psoriasis and PsA have greatly improved the QOL and function of patients with these disorders, but several major challenges remain to be addressed. A common feature underlying many of these challenges is the marked heterogeneity in presentation and clinical course, along with a limited understanding of disease-related pathways and key cellular and molecular mechanisms that initiate and perpetuate skin and musculoskeletal inflammation. GRAPPA comprises an international group of rheumatologists, dermatologists, methodologists, and patient research partners who have the ability and desire to form a large, cohesive CRN to address key unanswered questions that pertain to psoriatic disease, including: (1) how to align clinicians, patients, and investigators to optimize outcomes in psoriatic disease; (2) how to apply novel methods to psoriatic disease that take into account disease heterogeneity; and (3) how to develop precision medicine strategies to identify which patients will develop the disease, which are likely to progress from PsC to PsA, and which are likely to respond (or not) to a specific therapy. The CRN's vision and goals are outlined below, with a SWOT analysis and operational plan that includes financial projections. We anticipate that establishing this unique research network will provide an unparalleled opportunity to gain a more complete understanding of pathophysiologic mechanisms that will translate into improved therapies for patients with PsC and PsA.

2. Elevator pitch

- PsA is a prevalent heterogeneous disease that can lead to impaired function and decreased QOL. New therapies have greatly reduced inflammation and improved outcomes in PsA.

- Despite these advances, fewer than $30 \%$ of patients with PsA achieve remission. Additionally, over $40 \%$ of patients do not respond (defined as a $20 \%$ improvement) based on phase III clinical trial results. Many of those who do meet primary response criteria still have much residual inflammation. Further, divergent treatment responses in different domains (e.g., skin vs peripheral joints) within the same individual patients are not uncommon and many patients do not have access to these expensive medications.

- A better understanding of disease-related pathways will facilitate new drug development and improve treatment response.

- An international CRN of centers with PsC and PsA cohorts will facilitate the collection of a broad variety of biologic samples, thereby catalyzing scientific discovery, new drug development, and the identification of key disease biomarkers.

GRAPPA: The Group for Research and Assessment of Psoriasis and Psoriatic Arthritis; CRN: Collaborative Research Network; PsA: psoriatic arthritis; QOL: quality of life; PsC: cutaneous psoriasis; SWOT: strengths, weaknesses, opportunities, and threats.

7. Gisondi P, Tinazzi I, El-Dalati G, Gallo M, Biasi D, Barbara LM, et al. Lower limb enthesopathy in patients with psoriasis without clinical signs of arthropathy: a hospital-based case-control study. Ann Rheum Dis 2008;67:26-30.

8. Ciancio G, Ferracin M, Saccenti E, Bagnari V, Farina I, Furini F, et al. Characterisation of peripheral blood mononuclear cell microRNA in early onset psoriatic arthritis. Clin Exp Rheumatol 2017;35:113-21

9. Ogdie A, Langan S, Love T, Haynes K, Shin D, Seminara N, et al. Prevalence and treatment patterns of psoriatic arthritis in the UK. Rheumatology 2013;52:568-75.
Table 2. CRN mission, vision, and goals.

The CRN fits GRAPPA's mission

- GRAPPA's stated goals are (1) to promote the development of national and international collaborative registries of patients with PsA and PsC to standardize the data being obtained and to learn more about the natural history of the disease as well as its genomic underpinnings; (2) to work closely with representatives of member biopharmaceutical companies to promote and conduct research on effective therapies for PsA and PsC; and (3) to provide a forum for networking and communication between international researchers in rheumatology and dermatology, industry, PRP, and their organizations, and regulatory agencies. The establishment of a CRN addresses all of these goals.

1. Mission of the CRN

- To move precision medicine forward in psoriatic disease, PsA, and PsC.

2. $\mathrm{CRN}$ vision

- Align clinicians, patients, and investigators in improving outcomes in psoriatic disease.

- Apply novel methods to psoriatic disease that take into account disease heterogeneity.

- Develop precision medicine strategies by identifying patients who will develop psoriatic disease, progress from PsC to PsA, develop more severe PsA, and respond (or not) to a specific therapy.

3. CRN goals

- Short-term: (1) Identify and secure funding to support pilot collaborative projects and gain experience in processes and logistics; (2) establish a governance structure; (3) decide the structure of the bioresource (centralized or distributed) and decide which centers will be in the initial phase of the CRN; (4) establish SOP for collection and storage of samples; (5) decide where bioresources will be located; and (6) develop a formal structure for PRP engagement.

- Longer term: (1) Develop the infrastructure for data management (software, storage, staff, etc.); (2) establish policies for data sharing, publication, intellectual property, etc.; (3) collect samples in the network with local and central storage; (4) incorporate bioinformatics expertise; and (5) facilitate interaction between clinical centers and industry partners.

CRN: Collaborative Research Network; GRAPPA: The Group for Research and Assessment of Psoriasis and Psoriatic Arthritis; PsA: psoriatic arthritis; PsC: cutaneous psoriasis; PRP: patient research partner; SOP: standard operating procedures.

10. Eder L, Haddad A, Rosen CF, Lee KA, Chandran V, Cook R, et al. The incidence and risk factors for psoriatic arthritis in patients with psoriasis: a prospective cohort study. Arthritis Rheumatol 2016;68:915-23.

11. Nguyen UDT, Zhang Y, Lu N, Louie-Gao Q, Niu J, Ogdie A, et al. Smoking paradox in the development of psoriatic arthritis among patients with psoriasis: a population-based study. Ann Rheum Dis 2018;77:119-23.

12. Bowes J, Budu-Aggrey A, Huffmeier U, Uebe S, Steel K, Hebert $\mathrm{HL}$, et al. Dense genotyping of immune-related susceptibility loci reveals new insights into the genetics of psoriatic arthritis. Nat Commun 2015;6:6046.

13. Miyagawa I, Nakayamada S, Nakano K, Kubo S, Iwata S, Miyazaki $\mathrm{Y}$, et al. Precision medicine using different biological DMARDs based on characteristic phenotypes of peripheral $\mathrm{T}$ helper cells in psoriatic arthritis. Rheumatology 2019;58: 336-44. 
Strengths

- Sixty-three centers from around the world that are committed to participating.

- Centers have expertise in PsC and PsA, with large longitudinal cohorts to enable better-powered studies.

- Integrated development of research methods and SOP for sample collection.

- A strong and diverse network of highly motivated PRP.

- Track record of effective management and engagement of centers.

- Expertise among members in various aspects of psoriatic disease research, including clinical, basic, and translational research.

- Involvement of committed researchers at all career stages, including early, mid, and senior, as well as trainees.

Weaknesses

- International collaborations of this scale are complex because of differing laws and requirements in individual countries.

- Heterogeneous disease. Need full range of disease groups to fully understand outcomes.

- This effort will be expensive. Obtaining longterm, sustainable funding is a major challenge.

- Need for expertise in bioinformatics, big-data management, and computational biology.

- A major challenge to create and sustain a CRN of this magnitude.

Opportunities

- Much interest in developing ways to use existing therapies in a more sophisticated way in our patients and in identifying new therapeutic targets. The need is great.

- Interests from industry and IMI.

- Through advances in genetics, transcriptomics, epigenetics, proteomics, and metabolomics, we will likely uncover new pathways and biomarkers in psoriatic disease.

- Longitudinal course from PsC to PsA provides opportunity to intervene early and greatly alter or prevent the evolution to PsA.

- Large cohorts with the deep expertise this CRN provides will greatly accelerate discovery.

Threats

- Efforts at local centers to create bio-resources and longitudinal cohorts.

- Inability of some centers to collect data under established SOP.

- Challenges with IP, industry, and academic research centers in different countries.

SWOT: strengths, weaknesses, opportunities, and threats; PsC: cutaneous psoriasis; PsA: psoriatic arthritis; SOP: standard operating procedures; PRP: patient research partners; CRN: Collaborative Research Network; IMI: Innovative Medicines Initiatives; IP: intellectual property.

\section{Target stakeholders}

- Patients, physician investigators, and clinicians (including both dermatologists and rheumatologists), biopharmaceutical companies, private and public funding sources, and regulatory agencies.

2. Landscape analysis

- The need for a cooperative approach to invigorate translational research in PsA is evident, and the need is high.

- At this time, no major centers or collaborative efforts are under way to address the pertinent questions in PsC and PsA.

- A collaborative effort between academia, patients, and biopharmaceutical companies has been applied successfully to study other disorders through AMP, IMI, and RA-BioDAM.

3. Patient participation

- Over the last 5 yrs, GRAPPA has invested in the establishment of a structured network of informed PRP to provide the patient perspective in GRAPPA research initiatives. The CRN will use this resource of PRP to ensure that patient interests are considered when it comes to the prioritization of research topics, study design, raising of funds, CRN governance (as well as the associated data/biobanks and subworking groups), development of patient information and consent procedures, ethical discussions, and dissemination and implementation of CRN results.

4. Competition analysis and advantages of the CRN

- If we do not undertake this endeavor, it is highly likely that other efforts will arise that partner academics with pharma to address the critical questions in PsA. This may take place in many forms, including biopharmaceutical company-sponsored substudies attached to clinical trials, investigator-initiated studies, publicly funded research, and studies funded by private foundations. The major advantages of the GRAPPA-CRN over these other approaches are the expertise of the investigators, the large patient populations, and the development of validated SOP to ensure robust data collection and storage that will ensure that the results obtained are reproducible, credible, and valid.

CRN: Collaborative Research Network; PsA: psoriatic arthritis; PsC: cutaneous psoriasis; AMP: Accelerating Medicines Partnership; IMI: Innovative Medicines Initiatives; RA-BioDAM: Rheumatoid Arthritis Biomarkers for Joint Damage; GRAPPA: Group for Research and Assessment of Psoriasis and Psoriatic Arthritis; PRP: patient research partners; SOP: standard operating procedures. 
1. Strategic plan: The strategic plan will unfold in 5 phases:

- Phase 1: Identify funding sources initially to support pilot collaborative studies before more longterm funding support is secured and establish the overall structure and leadership of the CRN.

- Phase 2: Develop SOP and an infrastructure plan for data collection and storage. Address international barriers that inhibit or hinder collaboration and the sharing of data.

- Phase 3: Decide the major areas that the CRN will focus on. Three areas were identified at the meeting in the July 2017 GRAPPA-CRN meeting in Amsterdam, the Netherlands.

- Phase 4: Decide how the research effort will be applied: observational studies, clinical trial, data collection for a registry, etc.

- Phase 5: Begin data collection. To develop and test SOP at the various centers and establish the recruitment and data collection protocols, it may be advisable to start the process at only a few centers with experience in patient recruitment, sample preparation, and the storage and collection of patient-related data. Once the SOP and data collection processes are established and sample storage is deemed satisfactory, the network would be expanded.

2. Team

- GRAPPA Research Committee Members and other key people: C. Ritchlin, O. FitzGerald, D. Jadon, A.W. Armstrong, K. Callis Duffin, V. Chandran, D.D. Gladman, S. Pennington, C. Stober, M. de Witt (PRP), and D. O'Sullivan (PRP). We will also engage biopharmaceutical companies in this collaborative effort.

3. Operational plan

- Move forward with IMI and establish a license for GRAPPA in the United Kingdom and the European Union.

- Pursue other funding sources: NIH, AMP, private foundations, and philanthropic contributions.

- Decide on a nucleus of centers to participate in the initial phase of the CRN.

- Establish a leadership team and governance structure.

- Formalize and validate SOP.

- Establish infrastructure for the collection of data (location, software and database, personnel, etc.).

- Generate and obtain agreement for operations policies regarding data collection and sharing, publications, and other governance issues.

- Begin data collection and storage.

4. Financial projections

- Items to discuss include projected costs. The cost to support AMP is about US\$40 million, to cover 2 diseases over 5 yrs. We will need about $\$ 10$ million to get started, and that is with a limited number of sites. This type of support will require a substantial commitment over time. In the IPART Registry, each patient entered into the registry and followed for 2 yrs costs about CA $\$ 4800$, so this number will help with projections. Of course, these estimates may not directly apply to the CRN depending on the depth and scope of sample collection proposed. We will also have to estimate how many full-time staff will be required and how much support, if any, we can provide to the sites for coordinators and sample collection and storage.

5. Knowledge transfer, dissemination, and implementation

- A committee will be established to address these 3 critical areas comprising physicians, physician scientists, methodologists, and PRP. The CRN will hold standalone meetings and provide updates at the annual GRAPPA meeting and as part of GRAPPA updates at international dermatology and rheumatology meetings. A Website will be created that is accessible by all members of the CRN, which will contain validated SOP, recent manuscripts and presentations, and updated information on the number of samples collected in the network.

CRN: Collaborative Research Network; SOP: standard operating procedures; GRAPPA: Group for Research and Assessment of Psoriasis and Psoriatic Arthritis; PRP: patient research partner; IMI: Innovative Medicines Initiatives; US NIH: National Institutes of Health; AMP: Accelerating Medicines Partnership; IPART: International Psoriasis and Arthritis Research Team. 